\title{
Spontaneous Brillouin Scattering in Gas-filled Anti-resonant Fibre
}

\author{
Malak Galal ${ }^{1 *}$, Li Zhang ${ }^{1}$, Fan Yang ${ }^{1}$, Suneetha Sebastian ${ }^{1}$, Shoufei Gao ${ }^{2}$, \\ Yingying Wang ${ }^{2}$ and Luc Thévenaz ${ }^{1}$ \\ ${ }^{1}$ Ecole Polytechnique Fédérale de Lausanne, Group for Fibre Optics, 1015 Lausanne, Switzerland \\ ${ }^{2}$ Institute of Photonics Technology, Jinan University, Guangzhou 510632, China \\ *malak.galal@epfl.ch
}

\begin{abstract}
Spontaneous Brillouin scattering is measured for the first time in a hollow-core anti-resonant fibre (HC-ARF) filled with Nitrogen $\left(\mathrm{N}_{2}\right)$ gas at different pressures. A Brillouin gain of $0.029 \mathrm{~m}^{-1} \mathrm{~W}^{-1}$ is yielded at a pressure of 34.7 bar. (c) 2021 The Author(s)
\end{abstract}

\section{Introduction}

Hollow-core fibres are currently emerging quite fast owing to their remarkable capabilities in a wide range of applications. Recently, a sixfold Brillouin gain amplification over that observed in silica-core fibres was reported in gas-filled hollow-core photonic-crystal bandgap fibres (HC-PCFs), paving the way for the integration of hollowcore fibres in diverse fields [1,2]. State-of-the-art hollow-core anti-resonant fibres (HC-ARFs) [3] have recently gained increased attention due to their exceptionally low loss [4]. They have great potential to be utilised for understanding different fluid properties using nonlinear effects such as Brillouin scattering.

In this work, we present, for the first time to the best of our knowledge, the measurement of spontaneous Brillouin scattering in a $2 \mathrm{~m}$ long $\mathrm{N}_{2}$-filled 6-capillary HC-ARF at different pressures. A gain of $0.029 \mathrm{~m}^{-1} \mathrm{~W}^{-1}$ is yielded at a pressure of $34.7 \mathrm{bar}$, and a Brillouin linewidth of $12.8 \mathrm{MHz}$ which is roughly 3 times narrower than that obtained for a standard SMF.

\section{Experimental Setup}

The left figure of Fig. 1 shows the scanning electron micrograph (SEM) image of the fibre used in the experiment where $D$ is the diameter of the fibre core and it is equal to $32 \mu \mathrm{m}$. The setup utilised for the measurement of the Brillouin gain spectrum is depicted on the right figure of Fig. 1. The HC-ARF is filled with gas at different pressures which causes the occurrence of scattering just like in a standard single-mode fibre. The back-reflected light which passes from port 2 to port 3 reaches a balanced-detection photo-detector (PD) where heterodyne beating between the two branches occurs, and the Brillouin gain spectrum (BGS) for the whole fibre is acquired by an electrical spectrum analyser (ESA).

\section{Results and Discussion}

The light-sound interaction in the $\mathrm{N}_{2}$-filled HC-ARF resulted in a measured gain of $0.029 \mathrm{~m}^{-1} \mathrm{~W}^{-1}$ at a pressure of 34.7 bar. Additionally, the Brillouin gain spectrum has a linewidth of $12.8 \mathrm{MHz}$ which is roughly 3 times narrower than that of a standard SMF. Fig. 2 (a) shows a log-log graph highlighting the square dependence of
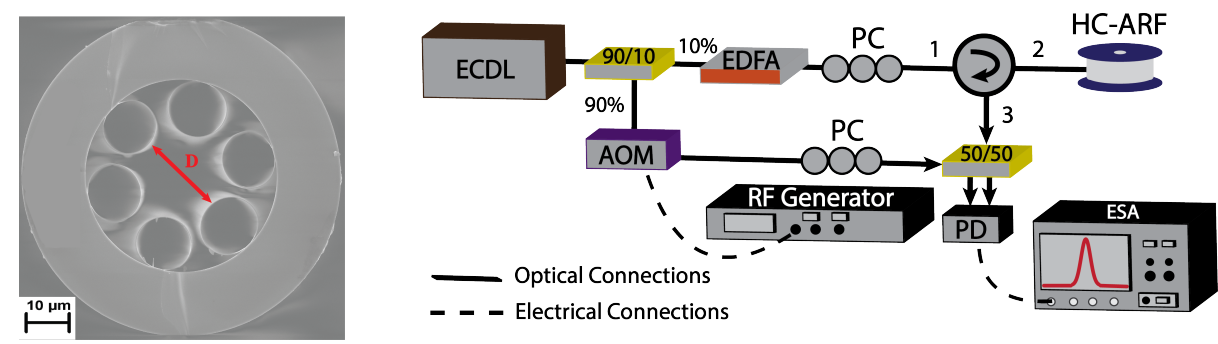

Fig. 1. Left figure: SEM image of the HC-ARF; Right figure: Experimental setup for the measurement of the Brillouin gain spectrum (BGS) (ECDL: External-cavity diode laser, EDFA: Erbium-doped fibre amplifier, PC: Polarization controller, AOM: Acousto-optic modulator.) 

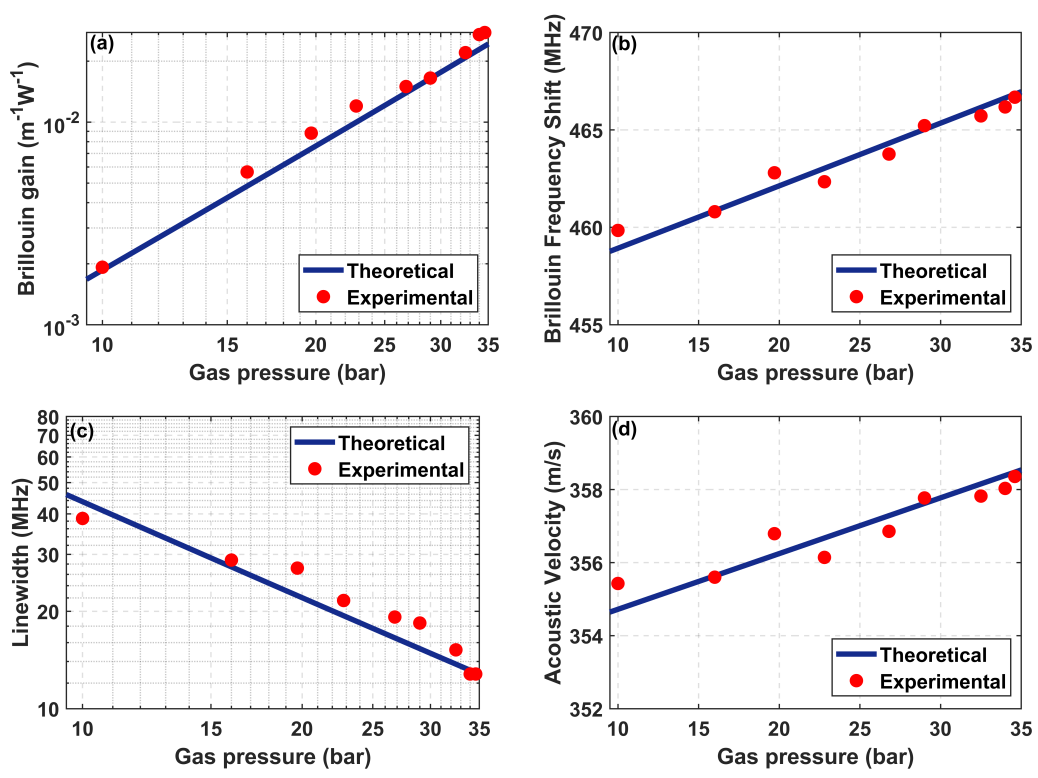

Fig. 2. Graphs showing different parameters as a function of the gas pressure: (a) Brillouin gain; (b) Brillouin frequency shift; (c) Brillouin linewidth; and (d) Acoustic velocity.

the Brillouin gain on the increase of the gas pressure which is reported in [1]. With larger pressure, the Brillouin frequency shift is also higher, and the Brillouin linewidth becomes narrower as can be observed on Fig. 2 (b) and (c), respectively. The acoustic velocity is then calculated from the measured Brillouin frequency shift through the phase matching condition of Brillouin scattering $\left(v_{B}=2 n_{\mathrm{eff}} v_{a} / \lambda\right)$, and is presented in 2 (d). The graphs in Fig. 2 clearly show that the experimental data is in great agreement with the theory. The theoretically calculated values for the Brillouin gain and linewidth can be calculated using the following expressions:

$$
\widetilde{g}_{B}=\frac{\gamma_{e}^{2} \omega^{2}}{n v_{a} c^{3} \rho \Gamma_{B} A_{\text {eff }}} \quad \text { (1) } \quad \Gamma_{B}=\frac{q^{2}}{\rho}\left(\frac{(\gamma-1) \kappa_{C}}{c_{p}}+\eta_{b}+\frac{4}{3} \eta_{s}\right)
$$

where $\gamma_{e}=\frac{\partial \varepsilon}{\partial \rho}$ is the electrostrictive coefficient in the gas medium, $\omega$ is the angular frequency of the light, $\rho$ is the gas density, $n$ is the gas refractive index, $c$ is the speed of light in vacuum, and $A_{\text {eff }}$ is the acousto-optic overlap effective area. $\Gamma_{B} / 2 \pi$ is the spectrum linewidth which is directly proportional to the acoustic attenuation $\Gamma_{B}$ given by Eq. (2), where $q$ is the acoustic wave-vector, $\eta_{s}$ and $\eta_{b}$ are the shear and bulk viscosities, respectively, $\kappa_{C}$ is the thermal conductivity, and $c_{p}$ is the specific heat capacity at constant pressure.

The Brillouin gain spectra were measured using a conventional rather simple reflectometer configuration (see right figure of Fig. 1) launching only the pump light into one fibre end without the need for a probe, and only onesided butt-coupling between the HC-ARF and a standard SMF was required. The results presented in this work show that the great potential of HC-ARF with its simple structure and its fairly large bandwidth is not merely limited to applications associated with communications and data transmission, but it can be quite useful for other purposes such as achieving gas Brillouin lasers or distributed fibre sensors that are only temperature-sensitive.

\section{Acknowledgements}

The authors acknowledge support from the Swiss National Foundation under grant agreement No. 178895 and 159897, and would like to thank Dr. Louise Jensen for capturing the SEM image of the HC-ARF.

\section{References}

1. F. Yang, F. Gyger, and L. Thévenaz, "Intense Brillouin amplification in gas using hollow-core waveguides," Nat. photonics 14, 700-708 (2020).

2. F. Gyger, "Brillouin scattering in gas-filled hollow-core fibres," Ph.D. thesis, École polytechnique fédérale de Lausanne (EPFL), Lausanne (2020).

3. S.-F. Gao, Y.-Y. Wang et al., "Bending loss characterization in nodeless hollow-core anti-resonant fiber," Opt. express 24, 14801-14811 (2016).

4. G. T. Jasion et al., "Hollow core NANF with $0.28 \mathrm{~dB} / \mathrm{km}$ attenuation in the $\mathrm{C}$ and L bands," in Optical Fiber Communication Conference, (Optical Society of America, 2020), pp. Th4B-4. 